\title{
Calcium Pantothenate
}

National Cancer Institute

\section{Source}

National Cancer Institute. Calcium Pantothenate. NCI Thesaurus. Code C74545.

The calcium salt of the water-soluble vitamin B5, ubiquitously found in plants and animal tissues with antioxidant property. Pentothenate is a component of coenzyme A (CoA) and a part of the vitamin B2 complex. Vitamin B5 is a growth factor and is essential for various metabolic functions, including the metabolism of carbohydrates, proteins, and fatty acids. This vitamin is also involved in the synthesis of cholesterol, lipids, neurotransmitters, steroid hormones, and hemoglobin. 